\title{
Ventajas y limitaciones sobre el uso del factor de impacto y las bases de datos del ISI como medidores de la actividad científica de un organismo público de investigación: análisis a partir de una experiencia y propuesta de indicadores alternativos
}

\author{
Advantages and limitations about the use of the impact factor and ISI databases to measure \\ the scientific research in a public research institution. Analysis from an alternative indicators proposal \\ Silvia Paz Otero (1), Rocío Herrerías Rubí (2), Miguel Ángel Alonso Valdivielso (3), \\ y Fernando Mérida Martín (4) \\ Instituto Nacional de Técnica Aeroespacial (INTA), Carretera de Ajalvir, Km. 4, 28850 Torrejón de Ardoz \\ (Madrid) (1) pazos@inta.es (2) herreriasrr@inta.es (3) alonsovm@inta.es (4) meridamf@inta.es
}

\begin{abstract}
Resumen
Con el objetivo de confeccionar indicadores de producción a partir de la recopilación de los currículos de los investigadores y partiendo de los datos obtenidos del estudio de producción científica del Instituto Nacional de Técnica Aeroespacial (INTA) para el período 2000-2005, en este trabajo se pretende analizar la validez del factor de impacto como medio para la evaluación de la actividad científica de los investigadores, así como reflexionar en torno a la propuesta de sistemas alternativos de medición, en particular el Índice $h$ de Hirsch, para tratar de definir cuál consideramos que sería más conveniente para nuestra institución de referencia.
\end{abstract}

Palabras clave: Instituto Nacional de Técnica Aeroespacial. Indicadores bibliométricos. Factor de Impacto. Producción científica. Evaluación científica.

\section{Introducción}

El Instituto Nacional de Técnica Aeroespacial "Esteban Terradas" (INTA) es un organismo público de investigación especializado en la investigación y desarrollo de tecnología aeroespacial. Fundado en la primavera de 1942 dentro del Ejército del Aire, pasó en 1977 a depender del recién creado Ministerio de Defensa, que integró en su estructura los antiguos Ministerios de las distintas Armas (Armada, Tierra y Aire). En la actualidad se inscribe dentro de la de la Secretaría de Estado de la Defensa.

\begin{abstract}
With the aim to create production indicators through the gathering of researchers curricula and starting with the data resulting from the INTA scientific production for the period 2000-2005, this paper analyzes the validity of the Impact Factor as a way to evaluate researchers' scientific activity. Also, a reflection is done about the proposal of alternative systems to measure the production, in particular the $\mathrm{H}$ index, from Hirsch, in order to define the most convenient system for our institution.
\end{abstract}

Keywords: National Institute of Aerospace Technique. Bibliometric indicators. Impact factor. Scientific production. Scientific evaluation.

Las funciones del INTA están definidas en su estatuto, aprobado en el año 2001. Entre ellas destacarían: la adquisición, mantenimiento y actualización de las tecnologías de aplicación en el ámbito aeroespacial; llevar a cabo ensayos, análisis y todo tipo de pruebas y trabajos experimentales para chequear, normalizar y certificar materiales, componentes, equipamiento, subsistemas y sistemas; actuar como laboratorio, centro tecnológico y servicio técnico para organismos públicos y la industria; diseminar el conocimiento científico y técnico para promover el desarrollo de la industria y la investigación 
tecnológica y científica; y proveer formación complementaria a los tecnólogos.

En la actualidad el INTA dispone de los siguientes centros:

- Torrejón de Ardoz (Madrid): Es la sede central donde se encuentran la mayor parte de los centros de investigación y grandes instalaciones del instituto.

- Centro de Experimentación de El Arenosillo -CEDEA-, (Huelva): Se encarga del, seguimiento, control, registro y evaluación de operaciones aeroespaciales

- Estación de Robledo de Chavela MDSCC (Madrid Deep Space Communication Complex): El complejo de Robledo de Chavela, denominado, forma parte de la red de espacio profundo de la NASA (DSN) y realiza seguimiento de misiones espaciales en órbita alrededor de la Tierra e interplanetarias (espacio profundo).

- Estación de Villafranca del Castillo (VILSPA): La estación espacial de Villafranca del Castillo está dedicada fundamentalmente al seguimiento y comunicaciones con naves de la Agencia Espacial Europea (ESA), a la que pertenece.

- Estación de Maspalomas (Islas Canarias): La estación espacial de Maspalomas está dedicada al seguimiento y comunicaciones espaciales de satélites. Sus funciones son el apoyo en misiones espaciales internacionales (seguimiento, telemetría y telecomando (TTC), monitorización y control, medida y calibración de Ranging, gestión de imágenes y datos, y la responsabilidad de la recepción y retransmisión de mensajes de alerta de balizas de emergencia, en la misión COSPASSARSAT.

- Centro de Ensayos de Granada: Controla y analiza la documentación, gestiona los ensayos en tierra y en vuelo, verifica la configuración de cada aeronave y determina las condiciones de seguridad en vuelo y las limitaciones de aeronavegabilidad.

- Centro de Astrobiología (CAB): Situado en la sede central del Instituto, se encuentra el Centro de Astrobiología, centro de carácter mixto compartido entre el Consejo Superior de Investigaciones Científicas (CSIC) y el INTA. Este centro se dedica al estudio del origen de la vida y su presencia en el Universo.

A lo largo de la dilatada historia del INTA el volumen de producción científica ha variado como resultado de la modificación de las estrategias productivas y de funcionamiento en las que el INTA ha estado inmerso.

En la actualidad el INTA se configura como un centro eminentemente tecnológico, manteniendo una sólida base científica a través de distintos laboratorios con un perfil de investigación básica, tales como el Centro de Astrobiología (CAB) y el Laboratorio de Astrofísica y Física Fundamental (LAEFF). El perfil tecnológico mencionado se encuentra presente en la multitud de laboratorios y dependencias diversas que se encargan de los ensayos y la certificación de distintas actividades o disciplinas como la electrónica, los materiales, la compatibilidad electromágnetica, la aerodinámica, la aeronavegabilidad, etc.

El resultado de tal actividad investigadora y tecnológica se refleja en la producción científica del INTA, que se encuentra en la línea media de las instituciones europeas similares al Instituto. Centros como el Laboratorio Nacional Aeroespacial de Holanda (NLR), el Centro de Investigación Aeroespacial (CIRA) de Italia, la Oficina Nacional de Estudios Aeroespaciales (ONERA) de Francia, ofrecen datos similares en cuanto a las publicaciones recogidas en las bases de datos científicas. Únicamente centros como el Laboratorio Aeroespacial de Alemania (DLR) muestra unas cifras manifiestamente superiores.

Los datos extraídos muestran que el índice de publicaciones no es elevado. Nuestra creencia es que esta circunstancia se debe al hecho de que todos estos centros, tienen, al menos en la actualidad, un perfil más tecnológico que científico, es decir, que vuelcan más su actividad en desarrollos tecnológicos que en investigación básica, siendo la producción científica generada menos proclive a aparecer en bases de datos científicas. Así mismo, en muchos casos son publicaciones opacas y de más complejo seguimiento e identificación.

\section{Análisis del estudio sobre la productividad del INTA (1990-2005)}

Tras la presentación el pasado año de los resultados de nuestro trabajo de investigación referido a la productividad del INTA y a la vista tanto de los resultados obtenidos como de las complicaciones derivadas del proceso de extrapolación de los mismos, se inició en nuestro departamento un período de reflexión basado en las conclusiones del mismo. Esta fase de análisis determinó la consideración inicial de que probablemente nuestras disciplinas de referencia (la Ingeniería Aeronáutica, la Astrobiología, etc.) y nuestro ámbito de aplicación de la investigación no aparecían suficientemente bien reflejados en 
las bases de datos del ISI. Si a ello unimos las dificultades constatadas para identificar y aislar los datos referidos a nuestra institución, se dieron las circunstancias adecuadas para que se iniciara el proceso de reflexión anteriormente mencionado, desde una doble perspectiva. Por una parte, la valoración del uso de fuentes de información e interrogación alternativas. Por otra, la consideración de otros sistemas de medición de la producción científica diferentes al Factor de Impacto.

Aunque presentados de forma independiente, ambos aspectos están íntimamente relacionados de manera que uno no se entiende sin hacer alusión al otro.

\subsection{Valoración del uso de fuentes}

de información alternativas

Las bases de datos del ISI, accesibles a través del portal Web of Knowledge (WOK) se consideran el medio de acceso a la información científica más recurrente en los estudios bibliométricos de producción científica. Su consideración como un medio pertinente de consulta le otorga entre la comunidad científica una cierta credibilidad en esa clase de estudios. Sin embargo, las críticas tampoco se mantienen al margen: en general, son fuentes de información en las que la producción científico-técnica de los países considerados periféricos no aparece lo suficientemente bien representada, debiendo recurrirse casi por obligación a otro tipo de fuentes de información complementarias para conseguir obtener la relación completa de las contribuciones firmadas por autores asociados a una institución académica.

En nuestro caso, recurrimos a otras bases de datos que consideramos pueden completar la información que tenemos, como ADS o SCOPUS. Con toda esa información, generamos una base de datos referencial bajo soporte Reference Manager v. 11. Las referencias se incorporan mediante descargas realizadas de las bases de datos multidisciplinares anteriormente comentadas. Estas descargas se completan con la información que nos proporciona regularmente la Unidad de Formación del Área de Recursos Humanos, a la que los investigadores deben entregar una copia de sus ponencias, aunque no de sus artículos.

No obstante, esta base de datos plantea problemas de diferente tipo, algunos muy obvios, como, por ejemplo, la identificación de los autores y las referencias citadas sólo por sus apellidos y por las iniciales de su nombre; una identificación que resulta insuficiente para aislar los datos, sobre todo en caso de los autores cuyos apellidos son excesivamente comunes. Otras consideraciones se refieren al ámbito casi exclusivamente anglosajón en el que extienden su ámbito de actuación estas bases de datos.

Otro de los problemas surgidos a raíz del estudio referenciado y que fue expuesto a modo de conclusión es que el motor de búsqueda no está lo suficientemente perfeccionado para filtrar selecciones complejas, al menos en el campo AD -address-, que es donde debemos interrogar al sistema para realizar análisis de dominio institucional.

$Y$ desde un punto de vista interno, no existe unanimidad en la adscripción de los autores a sus entidades de referencia. Éste es un elemento que debemos considerar y tratar de corregir a nivel interno impartiendo cursos de formación al personal investigador sobre pautas de publicación en revistas científicas, en aras de mejorar la calidad y la visibilidad de sus contribuciones.

\subsection{Consideración de otros sistemas de medición de la producción científica diferentes al factor de impacto}

El uso del Factor de Impacto (FI) generado por el Institute for Scientific Information (ISI) y publicado en el Journal Citation Reports (JCR) crece día a día no sólo entre la comunidad bibliométri$\mathrm{ca}$, sino también entre los investigadores y los gestores de políticas científicas (Bordons, 2002). El término fue acuñado en los años 60 para identificar el porcentaje de citas que reciben los artículos de una revista. El FI de una revista en un año es el número de citas que reciben los artículos publicados en esa revista en los dos años previos, dividido por el número de artículos publicados durante esos dos años. Los Fls son publicados anualmente en el JCR y aparecen claramente detallados en el portal WOK. Este portal ofrece, asimismo, un ranking de los artículos más citados por disciplinas identificadas previamente, los llamados "Hot papers". A pesar de que es considerado el recurso idóneo para acercarse a la producción científica de una materia, plantea problemas a la hora de interrogarla para acceder a la producción científica de una comunidad científica en particular, si ésta se escapa de los circuitos establecidos en el mundo anglosajón. El análisis del conocimiento generado en instituciones investigadoras y docentes situadas en países y regiones periféricas, se muestra incompleto si solamente consultamos las bases de datos del ISI. Estas bases de datos se alimentan, en un porcentaje muy elevado, de revistas y publicaciones del área anglosajona, y es un hecho constatado que muchos investigadores alejados de ese área 
encuentran todavía hoy una limitación idiomática relevante, puesto que la lengua de preferencia (por no decir, la exclusiva) es el inglés. Esta afirmación no debe ser considerada baladí, puesto en el caso español se ha constatado que frente a las bases de datos del ISI, para el estudio de la producción científica española en Ciencia y Tecnología, la base de datos ICYT de Ciencia y Tecnología desarrollada por el Centro de Información y Documentación Científica (CINDOC) del Consejo Superior de Investigaciones Científicas (CSIC) español, se ha mostrado mucho más eficaz y acorde con la realidad publicada, al incluir también entre sus referencias aquellas que utilizan el español como idioma de escritura y reflexión.

Junto con las limitaciones idiomáticas, el tipo de documentos a incluir constituye otro de los factores que limitan el número de referencias recuperadas y su inclusión en los estudios de productividad sobre una institución académicoinvestigadora de ámbito periférica. Las bases de datos del ISI sólo incluyen los artículos y ponencias consideradas relevantes por su FI, dejando fuera nuevamente numerosas contribuciones de otro tipo documental, aunque éstas hayan sido publicadas en revistas con $\mathrm{Fl}$. Pero es en el caso de los relatorios a congresos no considerados por el ISI en donde encontramos la principal laguna a la que nos debemos enfrentar cuando asumimos el análisis de una institución investigadora como la que nos ocupa, el Instituto Nacional de Técnica Aeroespacial. Su carácter eminentemente tecnológico y el carácter de sus investigaciones determinan también un cambio en los hábitos de publicación, al optar preferentemente por la asistencia a congresos y jornadas en los que compartir el conocimiento resulta más satisfactorio para la comunidad de investigadores de nuestro centro frente a la preferencia por la publicación en revistas.

Asimismo, se ha constatado que los trabajos aplicados (una buena parte de los que se publican en nuestro centro como corresponde a un centro de perfil más tecnológico que científico) encuentran una mejor acogida de cara a su publicación y difusión en los seminarios que en revistas especializadas.

Estableciendo una comparativa entre el número de ponencias firmadas por autores del INTA y registradas tanto en ISI como en nuestra base de datos (generada, recordemos a partir de las comunicaciones que nuestros investigadores deben entregar en la biblioteca al asistir a un congreso) tomando como referencia el año 2005, las diferencias son notorias. En la biblioteca se dispone de 115 ponencias, de las cuales sólo 6 aparecen recogidas en el ISI. Una reflexión en torno a la disparidad de las cifras nos lleva claramente a plantearnos la consideración no ya del uso de fuentes de información alternativas, sino también de otros indicadores que nos permitan medir y parametrizar con más eficiencia la carrera investigadora de nuestros científicos, que es uno de nuestros objetivos prioritarios.

En la literatura especializada, y fruto quizás del desencanto por la utilización del $\mathrm{FI}$, surgieron diferentes indicadores que pretendían corregir ciertas desviaciones apreciadas en su uso. Entre ellos, podemos citar los siguientes:

- Factor de Impacto Ajustado (Adjusted IF), que calcula el cómputo de las citas en un período de cuatro años (Ramírez, 2000). Si tenemos en cuenta que el FI sólo calcula las contribuciones en dos años, establecer un margen temporal mayor nos podría proporcionar un acceso más razonable a las publicaciones. El problema de aplicabilidad en nuestro centro es la propia política de publicación imperante, que sitúa el plazo medio de publicación de los resultados de una investigación en un período superior a los cuatro años fijados.

- Cited half-life IF, que se obtiene dividiendo el cómputo de las citas en relación con la vida media de citación de la publicación (Sombatsompop, 2004). Surge de la consideración de que el cálculo teniendo en cuenta sólo los dos últimos años no es ni significativo ni extrapolable a realidades diferentes.

- El Eurofactor (EF) se presentó en el año 2002 como un factor alternativo al $\mathrm{FI}$ respondiendo a la demanda de la comunidad científica europea que no se consideraba excesivamente bien representada en las bases de datos del ISI. Se incluyeron en la primera edición del mismo un total de 500 publicaciones incluidas según criterios de calidad de las mismas. El EF se obtiene dividiendo el número de citas de una revista por el EF-coefficient multiplicando por la raíz cuadrada de la suma del número de citas y del número de artículos (Hofbauer, 2002; Aleixandre-Benavent, 2007). En nuestro caso, la tendencia de publicación constatada es preferir su inclusión en revistas anglosajonas (americanas, en su mayor parte) por lo que tampoco estamos seguros de su correcta aplicación para analizar la producción científica de nuestro centro.

- Factor de Impacto Ponderado por especialidad (Disciplinary IF), destinado a establecer parámetros de comparación entre las publicaciones de áreas y disciplinas científicas distintas, se obtiene dividiendo el FI de cada revista entre el FI máximo de su área, que corresponde a la primera publicación del área con 
mayor FI (Ramírez, 2000). Podría ser utilizado para calcular el FI de nuestro centro, pero entramos en la disyuntiva de tener que adscribir nuestras publicaciones a las categorías temáticas ISI, con lo que ello conlleva: en nuestro estudio del año 2006 constatamos que un gran número de trabajos aparecen encuadrados dentro de la categoría "Física", siendo contribuciones que identificamos dentro de la materia de la astronomía, por lo cual consideramos que hay una inapropiada ubicación de categorías, al menos en este caso.

- Journal to field impact store (JFIS), que se basa en cuatro tipos de documentos (artículos, cartas, notas, revisiones) y en el cálculo del $\mathrm{FI}$ a partir de esos cuatro elementos, tanto en el numerador como en el denominador (Leeuwen, 2002). El impacto de una revista es comparado con el impacto que ostenta la disciplina a la que pertenece, en base a las materias diseñadas en las bases de datos del ISI. La obligación de establecer una comparativa con disciplinas ISI vuelve a ser uno de los factores determinantes para descartar su aplicación en nuestro centro.

\subsection{El factor $h$ de Hirsch}

Mención aparte merece el factor $\mathrm{h}$. A pesar de ser relativamente reciente (su aparición es en el año 2005), ha generado numerosa bibliografía, y sus partidarios se muestran tan fervientes como sus detractores.

El factor $h$ fue desarrollado y presentado por Jorge E. Hirsch en el año 2005. Su ideólogo es un físico americano, que harto de comprobar cómo su disciplina de investigación era, según él, injustamente tratada en las listas anuales del $\mathrm{FI}$, desarrolló un índice capaz de evaluar no las revistas en las que un científico publica, sino su propia carrera investigadora y profesional. Este índice (denominado "índice h", como su creador) consiste en el número de artículos que tiene un autor con tantas o más citas que su factor $h$. Las ventajas de este indicador son, entre otras (Valderrama-Zurián, 2007): a) es un promedio extendido a lo largo de los años que sólo puede aumentar o mantenerse estancado; b) se aplica a científicos individuales y permite comparar carreras de científicos de diferentes edades; c) permite extrapolar el rendimiento de un científico a medio plazo.

En lo que se refiere a su aplicación para el análisis de dominio institucional, debemos decir que entre sus ventajas encontramos también sus desventajas, puesto que en su definición no se indica nada sobre su aplicación al análisis de instituciones. De hecho, su esencia radica en realizar análisis individuales de la producción de los científicos y, a través de esos análisis, extrapolar datos a la comunidad global a la que pertenece ese científico, ya sea por disciplinas o por entidades.

A nivel práctico, hemos realizado una prueba de aplicación del índice $\mathrm{h}$ tomando como referencia el Centro de Astrobiología (CAB) para el período 2000-2005. El Centro de Astrobiología (CAB), centro mixto CSIC-INTA, está ubicado en el campus del Instituto Nacional de Técnica Aeroespacial (INTA), en Torrejón de Ardoz. Es el primer centro de investigación no estadounidense asociado al NASA Astrobiology Institute (NAI), que reúne a catorce grupos de científicos y laboratorios dispersos por la geografía de los Estados Unidos de América. Los miembros que forman parte del NAI son: Arizona State University, Carnegie Institution Washington, Harvard University, NASA Ames Research Center, NASA Jet Propulsión Laboratory, NASA Jonson Space Center, Pennsylvania State University, Michigan State University, The Scripps Research Institute, University of California Los Angeles, University of Colorado, University of Rhode Island, University of Washington y Woods Hole Marine Biological Laboratory.

La Astrobiología como tal es una ciencia relativamente nueva y por su idiosincrasia no está recogida en las diferentes bases de datos bibliográficas, no existiendo por descontado una base de datos específica. Desde el punto de vista documental podríamos decir que la Astrobiología es una especie de metadisciplina, formada por las disciplinas que mostraremos a continuación tras tratar de determinar la presencia de la Astrobiología en el ISI, entre los años 2000 y 2005.

El número de artículos obtenidos es de 941 repartidos en 91 materias, lo que da una idea de la transdisciplinariedad de la materia que nos ocupa, y la idoneidad de la base de datos ISI para un trabajo de estas características. Dentro de estas 91 materias podemos destacar un núcleo principal formado por 5 disciplinas ISI, todas ellas con más de 100 artículos, y un segundo núcleo formado por 17 disciplinas con más de 10 artículos. Un total de 22 disciplinas se reparten 1.202 artículos. Las 68 disciplinas restantes se reparten un total de 214 artículos (Tabla 1).

Los resultados obtenidos en la tabla 1 ponen de manifiesto la dificultad de evaluar de forma genérica los autores y las instituciones a las que representan teniendo en cuenta las citas recibidas, puesto que de forma análoga a su comportamiento particular fuera de la Astrobiología las culturas de citación varían enormemente de una 
disciplina a otra, repercutiendo igualmente en su índice h (gráfico 1). El trabajo de Hirsch lo pone de manifiesto al comparar la Física y la Biología. Las diferencias están causadas por la dependencia de $\mathrm{h}$ del tamaño de la población que potencialmente puede citar el trabajo: si son muchos los investigadores trabajando en una materia, las citas pueden ser mayores que en una comunidad científica pequeña.

En cuanto a los patrones de citación, hemos observado que de los 941 artículos, tan sólo 239 no tienen ninguna cita. Cerca del $77 \%$ han sido citados alguna vez, lo que da una idea de la visibilidad de la Astrobiología, y que los parámetros de citación difieren del resto de disciplinas. Estructurados por tipología documental, obtenemos los siguientes datos: Se los 941, 705 son artículos: y, de éstos, 607 han sido citados en alguna ocasión, con un promedio de citas de 10,58 y un índice-h 38; 90 son "Meeting Abstract".
Sólo 5 tienen citas, con un promedio de citas de 0,09 y un índice-h 1; 59 son "Review". Sólo 4 no tienen citas, con un promedio de citas de 22,27 y un índice-h 20; 45 son "Material Editorial". 26 tienen citas, con un promedio de citas de 6,18 y un índice-h 9; y 12 son "Letter". Sólo 5 tienen citas, con un promedio de citas de 2,50 y un índice-h 4.

Por lo tanto, debemos considerar que el Índice $\mathrm{h}$ resulta adecuado para analizar las carreras de las investigadores a nivel individual, pero no está lo suficientemente desarrollado para ser aplicado ni en materias complejas ni en el análisis de un dominio como el que nos ocupa. A su favor tiene el hecho que tiene en cuenta todo tipo de publicaciones, aunque son los "reviews" y los "artículos" los que aumentan la visibilidad y el promedio de citas (Gráfico 1, en la página siguiente).

\begin{tabular}{lrrcc}
\hline Materia & Artículos & Citas & Promedio de citas por artículo & Índice- $h$ \\
Astronomy \& Astrophysics & 317 & 2529 & 7,98 & 23 \\
Geosciences, Multidisciplinary & 160 & 511 & 3,19 & 11 \\
Multidisciplinary Sciences & 116 & 1901 & 16,39 & 24 \\
Geochemistry \& Geophysics & 113 & 685 & 6,06 & 15 \\
Biology & 108 & 479 & 4,44 & 12 \\
Biochemistry \& Molecular Biology & 44 & 802 & 18,23 & 15 \\
Microbiology & 39 & 551 & 14,13 & 13 \\
Engineering, Aerospace & 36 & 56 & 1,56 & 4 \\
Genetics \& Heredity & 32 & 908 & 28,38 & 17 \\
Physics, Multidisciplinary & 31 & 205 & 6,61 & 7 \\
\hline
\end{tabular}

Tabla I. Patrones de citación por materias en Astrobiology

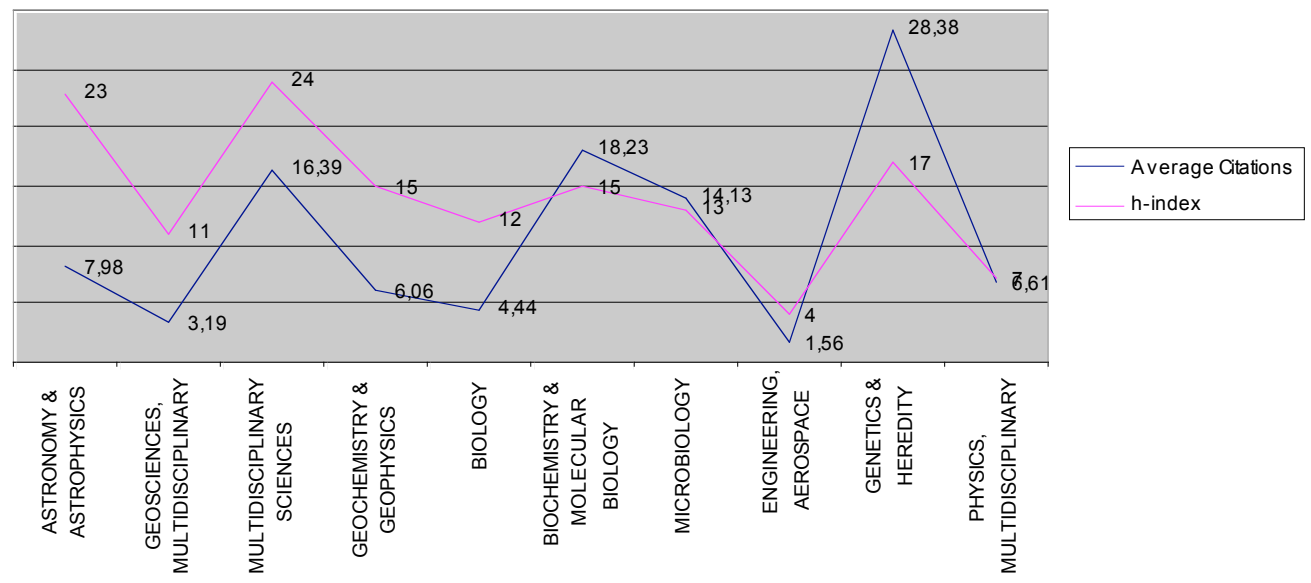

Gráfico 1. Patrones de citación por materias en Astrobiology (2000-2005). 


\section{Conclusiones}

1. Para la evaluación de la producción científica de un centro de investigación aeroespacial, la única y exclusiva consulta de las bases de datos del ISI resulta insuficiente.

2. La obtención de indicadores de productividad debe realizarse combinando distintas fuentes de información, con el fin de obtener una información lo menos sesgada posible.

3. En sintonía con otros estudios similares, debe considerarse muy positivamente la consulta de la base de datos YCIT, en la que la producción española de Ciencia y Tecnología aparece más ampliamente referenciada.

4. La mayoría de las iniciativas surgidas como crítica al JCR, parten de los datos recopilados en las bases de datos del ISI, por lo que los datos referidos a nuestra institución no serán nunca completos.

5. La utilización del índice $\mathrm{h}$ debe considerarse para la valoración de las carreras individuales de los científicos, pero resulta un poco complicado extrapolarlo a la valoración de una institución en su conjunto.

6. Las bases de datos del ISI no han demostrado ser lo suficientemente eficaces para el análisis de metadisciplinas, como sucede en el caso de la Astrobiología que está muy representada en nuestra institución.

7. En general, podemos afirmar que para realizar un análisis de la producción científica del Instituto Nacional de Técnica Aeroespacial debemos recurrir a la confección de bases de datos e indicadores propios que resulten de la combinación de diferentes elementos, con el fin último de obtener una información lo completa y ajustada a la realidad.

\section{Referencias}

Aleixandre-Benavent, Rafael; Valderrama-Zurián, Juan Carlos; González-Alcaide, Gregorio (2007). El factor de impacto de las revistas científicas: limitaciones e indicadores alternativos. // El profesional de la información. 16:1 (2007) 4-11.

Bakkalbasi, Nisa; Bauer, Kathleen; Glover, Manis; Wang, Lei (2006). Three options for citation tracking: Google Scholar, Scopus and Web of Science. // Biomedical Digital Libraries. 3:7 (2006). http://bio-diglib.com/content/3/1/7 (2007-06-12).

Barendse, William (2007). The strike rate index: a new index for journal quality based on journal size and the h-idex of citations. // Biomedical Digital Libraries. 4:3 (2007). http://bio-diglib.com/content/4/1/3 (2007-06-12).

Bollen, Johan; Sompel, Herbert van de; Smith, Joan A.; Luce, Rick (2005). Toward alternative metrics of journal impact: a comparison of download and citation data. // Information Processing and Management. 41 (2005) 1419-1440.

Burnham, Judy (2006). Scopus database: a review. // Biomedical Digital Libraries. $3: 1$ (2006). http://biodiglib.com/content/3/1/1 (2007-06-12).

Dong, Peng; Loh, Marie; Mondry, Adrian (2005). The "impact factor" revisited. // Biomedical Digital Libraries. 2:7 (2005) http://www.bio-diglib.com/content/2/1/7 (2007-0513).

Grupo Scimago (2007) El índice h de Hirsch: su aplicación a algunos de los científicos españoles más destacados. // El profesional de la información. 16:1 (2007) 47-49.

Hirsch, Jorge E. (2005). An index to quantify and individual's scientific research output. Proceedings of the National Academy of Sciences of the USA. 102 (2005). http://arxiv.org/abs/physics/0508025 (2007-03-07).

Hofbauer, R.; Frass, M.; Gmeiner, M.; Kaye, A. D. (2002). Euro-Factor (EF)TM. The European Journal Quality Factor. The new European "scientific currency". Viena: Vicer Publishing, $2002 . \quad$ http://www.univlille1.fr/lea/Menu du Site/Publications/Acrobat/VICEREUROFACTOR.pdf (2007-04-13).

Kaltenborn, K. F.; Kuhn, K. (2004). The journal impact factor as a parameter for the evaluation of researchs and research. // Revista Española de Enfermedades Digestivas. 96:7 (2004) 460-476.

Leewen, T. N. van; Moed, Henk F. (2002). Development and application of journal impact measures in the Dutch science system. // Scientometrics. 53:2 (2002) 249-266.

Moed, H. F.; Leeuwen, T. van; Reedijk, J. (1999). Towards aproppiate indicators of journal impact. // Scientometrics. 46:3 (1999) 575-589.

Rousseau, Ronald (2005). Median and percentile impact factors: a set of new indicators. // Scientometrics. 63:3 (2005) 431-441.

Sombatsompop, Narongrit; Markpin, T.; Premkamolnetr, N. (2004). A modified method for calculating the Impact Factor of journals in ISI Journal Citation Reports: Polymer Science Category in 1997-2001. // Scientometrics. 60:2 (2004) 217-235.

Sombatsompop, Narongrit; Markpin, T.; Buranathiti, T.; Ratchatahirun, P.; Metheenukul, T.; Premkamolnetr, N.; Yochaim, W. (2007). Categorization and trend of materials science research from Science Citation Index (SCl) database: a case study of ceramics, metallurgy, and polymer subfields. // Scientometrics. 71:2 (2007) 283-302.

Verbeek, Arnold; Debackere, Koenraad; Luwel, Marc; Zimmerman, Edwin (2002). Measuring progress and evolution in science and technology. I: The multiple uses of bibliometric indicators. // International Journal of Management Reviews. 4:2 (June 2002) 179-211.

Verbeek, Arnold; Debackere, Koenraad; Luwel, Marc; Zimmerman, Edwin (2002). Measuring progress and evolution in science and technology. II: The multiple uses of technometric indicators. // International Journal of Management Reviews. 4:3 (September 2002) 213-231.

Whitehouse, G. H. (2002). Impact factors: facts and myths. // European Radiology. 12 (2002) 715-717. 